\title{
Base de dados atualizados de indicadores padronizados de citações de autores científicos na área de equoterapia: uma revisão bibliométrica
}

\author{
Standardized database of standardized indicators of citations of scientific authors in the field of \\ hippotherapy: a bibliometric review \\ Base de datos estandarizada de indicadores estandarizados de citas de autores científicos en el \\ campo de la hipoterapia: una revisión bibliométrica
}

Recebido: 22/02/2021 | Revisado: 28/02/2021 | Aceito: 29/03/2021 | Publicado: 06/04/2021

Giselda Tavares de Araujo

ORCID: https://orcid.org/0000-0002-5101-5750

Universidade Federal do Triângulo Mineiro, Brasil

E-mail: giseldatavares05@gmail.com

Leonardo Augusto Lombardi

ORCID: https://orcid.org/0000-0002-9735-8804

Universidade Federal do Triângulo Mineiro, Brasil

E-mail: nda_leo@yahoo.com.br

Luis Fernando Santana

ORCID: https://orcid.org/0000-0001-6615-975X Instituto Federal de Educação, Ciência e Tecnologia do Triângulo Mineiro, Brasil E-mail: luisfernando@iftm.edu.br

Fernando Seiji Silva

ORCID: https://orcid.org/0000-0002-6373-8817 Universidade Federal do Triângulo Mineiro, Brasil

E-mail: fernando.silva@uftm.edu.br

Daniel Ventura Dias

ORCID: https://orcid.org/0000-0001-8113-8228 Universidade Federal do Triângulo Mineiro, Brasil

E-mail: daniel.dias@uftm.edu.br

Ana Paula Espindula

ORCID: https://orcid.org/0000-0002-9282-4482 Universidade Federal do Triângulo Mineiro, Brasil

E-mail: ana.espindula@uftm.edu.br

\begin{abstract}
Resumo
Equoterapia é um método terapêutico que utiliza o cavalo como ferramenta de tratamento em diversos comprometimentos físicos e intelectuais, complementando a reabilitação física e/ou social e promovendo resultados funcionais. Trata-se de um estudo descritivo e quantitativo, que teve como objetivo realizar mapeamento científico sobre a equoterapia, durante todo o período, por meio da análise bibliométrica. A busca apresentou resultados de 1972-2020, foram encontrados 360 artigos, 945 palavras chaves, 1.265 autores, com média de 3,37 por documento e 4,06 para coautores. A média de publicação foi 5,32 totalizando 12.532 referências. A análise permitiu descrever as palavras chaves mais citadas, fontes mais relevantes, publicação científica anual, autores mais citados, autores mais relevantes, estudos mais citados localmente, estudos mais citados globalmente, classificação de periódicos segundo fator de impacto e países mais citados. Promovendo mapeamento científico sobre a equoterapia e contribuindo para pesquisas futuras.
\end{abstract}

Palavras-chave: Terapia assistida por cavalos; Base de dados; Revisão; Bibliometria.

\begin{abstract}
Hippotherapy is a therapeutic method that uses the horse as a treatment tool for various physical and intellectual impairments, complementing physical and/or social rehabilitation and promoting functional results. It is a descriptive and quantitative study, which aimed to perform a scientific mapping on hippotherapy, throughout the period, through bibliometrics analysis. The search showed results from 1972-2020, 360 articles were found, 945 keywords, 1.265 authors, with an average of 3,37 authors per document and 4,06 for co-authors. The average publication was 5,32 with a total of 12.532 references. The analysis allowed describing the most cited keywords, most relevant sources, annual scientific publication, most cited authors, most relevant authors, most cited studies locally, most cited studies globally, classification of journals according to the impact factor and most cited countries. Promoting a scientific mapping on hippotherapy and thus contributing to future research.
\end{abstract}


Keywords: Equine-assisted therapy; Database; Review; Bibliometrics.

\section{Resumen}

La equitación es un método terapéutico que utiliza al caballo como herramienta de tratamiento de diversas deficiencias físicas e intelectuales, complementando la rehabilitación física y / o social y promoviendo resultados funcionales. Se trata de un estudio descriptivo y cuantitativo, que tuvo como objetivo realizar un mapeo científico de la hipoterapia, a lo largo del período, mediante análisis bibliométrico. La búsqueda arrojó resultados de 1972-2020, se encontraron 360 artículos, 945 palabras clave, 1.265 autores, con un promedio de 3.37 autores por documento y 4.06 para coautores. La publicación promedio fue de 5.32, totalizando 12.532 referencias. El análisis permitió describir las palabras clave más citadas, fuentes más relevantes, publicación científica anual, autores más citados, autores más relevantes, estudios más citados a nivel local, estudios más citados a nivel mundial, clasificación de revistas según el factor de impacto y países más citados. Impulsar un mapeo científico sobre hipoterapia y contribuir así a futuras investigaciones.

Palabras clave: Terapia asistida por caballos; Base de datos; Revisión; Bibliometría.

\section{Introduçãa}

A equoterapia é um método terapêutico que utiliza o cavalo para promover ganhos a nível físico e psíquico, o que contribui para um desenvolvimento biopsicossocial de pessoas com necessidades especiais e/ou déficit neuromotor (Ande, 2021).

O método se dá pela interação cavalo-humano, e é usado como uma intervenção complementar, alternativa ou integrativa que incorpora experiências físicas e psicossociais que facilitam o processo de tratamento e/ou reabilitação, o que pode proporcionar melhora na saúde física, psicológica ou social do indivíduo (White-lewis et al., 2019). Há registros, desde os tempos antigos, sobre o uso dos animais para tratamentos de diversas doenças. De acordo com (Althobaiti et al., 2019), o primeiro reconhecimento do cavalo como um agente de cura pode ser encontrado na mitologia primitiva. Hipócrates, frequentemente conhecido como pai da medicina, descreveu sobre o "ritmo de cura da equitação" (Pálsdóttir, Gudmundsson \& Grahn, 2020) os animais passaram a ser usados terapeuticamente no cuidado de soldados durante a primeira e segunda Guerras Mundiais, e foram agentes terapêuticos no tratamento e cuidados, minuciosamente, na década de 1950, onde a fisioterapeuta norueguesa Elspeth Bödker tornou-se pioneira na reabilitação de crianças com poliomielite, paralisia cerebral e outras doenças neurológicas. De acordo com a American Hippotherapy Association (Aha, 2021), a equoterapia é uma ferramenta para envolver os sistemas sensoriais, neuromotores e cognitivos a fim de promover resultados funcionais.

O método terapêutico utiliza a marcha e os movimentos naturais do cavalo para fornecer estímulos motores e sensoriais (Koca \& Ataseven, 2016) Tais estímulos podem estar relacionados a mudanças na bioquímica humana, linguagem corporal ou entonações vocais (Path-Learn, 2020). Uma necessidade física, cognitiva ou emocional não impede uma pessoa de interagir com cavalos (Path-Learn, 2020), o que o torna uma opção de tratamento para diversas condições e/ou doenças clínicas, além de ser uma atividade promotora de saúde e que, consequentemente, pode reduzir custos (Pálsdóttir, Gudmundsson \& Grahn, 2020).

Atualmente, em todo o mundo, existem vários programas de equoterapia (Russell, 2013). No Brasil, atualmente são realizados quatro programas: hipoterapia; educação/reeducação; pré-esportivo e prática esportiva paraequestre. Na hipoterapia o cavalo é inserido como um instrumento fisioterapêutico, enquanto no programa de educação o mesmo é inserido como um instrumento pedagógico. O programa pré-esportivo é realizado a partir da promoção de uma realidade social por meio do animal, e no paraequestre o cavalo é promotor da inserção social por meio do esporte (Ande, 2021). A Professional Association of Therapeutic Horsemanship International, relata que para-equestres amputados competiram com sucesso; crianças com transtorno de déficit de atenção aprenderam a controlar o cavalo, adquiriram independência e trabalho em equipe, assim como as crianças com transtorno de autismo (Path-Learn, 2020). 
Estudos mostram também melhora na postura em crianças com paralisia cerebral (Matusiak-wieczorek, Dziankowska-zaborszczyk \& Borowski, 2020) redução da atividade mioelétrica dos músculos mastigatórios em idosos (De mello et al., 2020); redução da dor e melhora da amplitude de movimento e qualidade de vida de adultos e idosos com artrite (Campos, 2007; White-Lewis, 2020) melhora nos aspectos psicológico, comportamental, social e motor e na postura de crianças com síndrome de Down (Campos, 2007; Espindula et al., 2016) e melhora do equilíbrio e flexibilidade em idosos (Diniz et al., 2020). Todas as atividades equoterápicas realizadas são baseadas em fundamentos técnicos científicos, e são desenvolvidas por equipe multiprofissional, envolvendo profissionais da saúde, educação e equitação (Ande, 2021), o que demanda atualização científica constante em todas as áreas envolvidas.

Sendo assim, esse estudo justifica-se pela crescente necessidade da prática baseada em evidências, tendo como objetivo realizar uma análise de mapeamento sobre a produção científica na área de equoterapia, durante todo o período, e analisar os principais autores, fontes de publicação e artigos mais citados na área, proporcionando o mapeamento da ciência na equoterapia e contribuindo com um conjunto amplo de informações científicas sobre a área em questão.

\section{Metodologia}

Trata-se de um estudo descritivo e quantitativo. As pesquisas foram realizadas por meio do portal da CAPES/MEC, em quatro bases de dados bibliográficas: Web of Science, Scopus, PubMed e Cochrane no dia 13/10/2020. Em seguida, foi utilizado o software R Studio para realizar o mapeamento e a ferramenta Biblioshiny, por meio do Bibliometrix, para a análise bibliométrica. A realização de análise abrangente de mapeamento científico bibliometrix suporta um fluxo de trabalho recomendado para realizar análises bibliométricas. Como é programado em $\mathrm{R}$, a ferramenta proposta é flexível e pode ser rapidamente atualizada e integrada com outros pacotes R estatísticos. (Aria \& Cuccurullo, 2017; Pereira et al., 2018; Baldam, 2021) As palavras chaves utilizadas para a busca nas bases de dados foram: "Hippotherapy" OR "Equine-Assisted Therapy " OR "Therapeutic Horseback Riding".

\section{Resultados}

Todas as informações foram obtidas a partir do software R Studio com a ferramenta bibliometrix. A busca, realizada com o filtro durante o período de 1972 à 2020, resultou em 375 documentos, com uma média de publicação de 5,32 e 12.532 referências. Foram encontrados 360 artigos, 945 palavras chaves, 1.265 autores, com uma média de 3,37 autores por documento, 4,06 para coautores e 1.557 citações por documento (Tabela 1).

Tabela1. Principais informações encontradas na pesquisa.

\begin{tabular}{ll}
\hline Período & $\mathbf{1 9 7 2}$ à 2020 \\
Documentos encontrados & 375 \\
Média anual de publicação & 5,32 \\
Artigos & 360 \\
Palavras chaves & 945 \\
Autores & 1.265 \\
Média de autor por documento & 3,37 \\
Média de coautor por documento & 4,06 \\
Média de citação por documento & 1.557 \\
Total de referências & 12.532 \\
\hline
\end{tabular}

Fonte: Autores (2020). 
As 10 palavras chaves mais citadas foram: Crianças; Hipoterapia; Paralisia cerebral; Agroecologia; Marcha; Equilíbrio; Terapia; Função motora grossa; Movimento social e Exercício (Figura 1).

Figura 1. Representação das palavras chaves mais relevantes utilizadas na pesquisa.

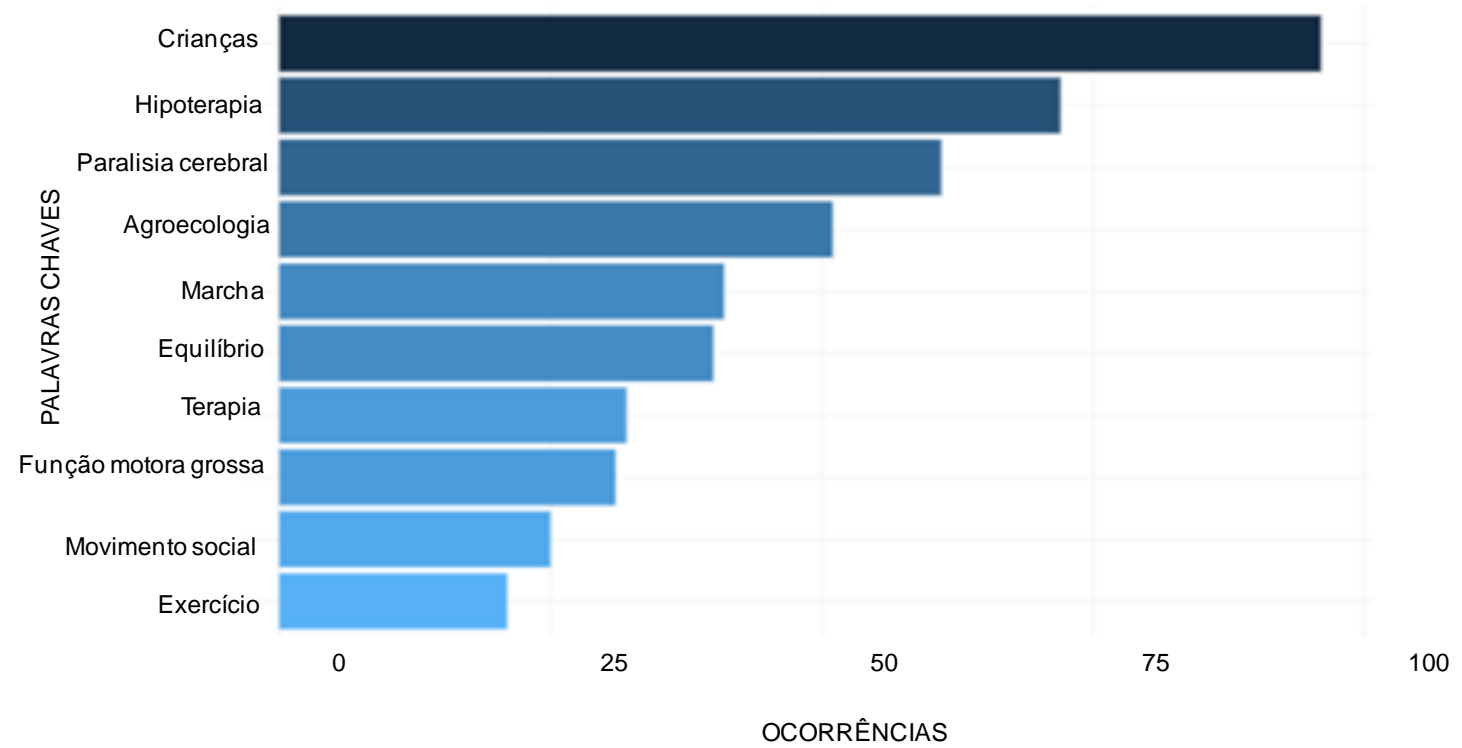

Fonte: Autores (2020).

Dentre todas as fontes científicas mundiais, 20 revistas foram consideradas as mais relevantes na área, devido ao maior número de publicações de artigos em seus periódicos, com destaque para o Journal of Physical Therapy Science, com maior número de manuscritos publicados (Figura 2).

Figura 2. Representação das fontes mais relevantes encontradas na pesquisa.

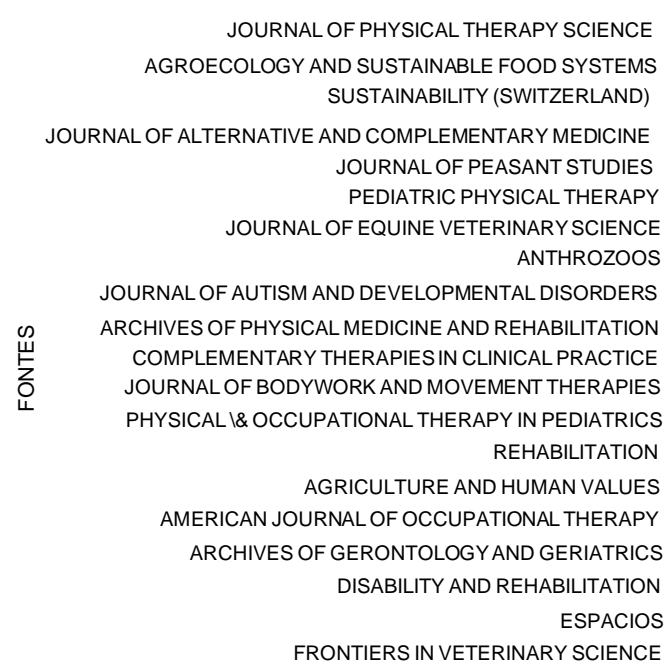

JOURNAL OF PHYSICAL THERAPY SCIENCE SUSTAINABLE FOOD SYSTEMS AND COMPLEMENTARY MEDICINE JOURNAL OF PEASANT STUDIES NARY SCIENCE ANTHROZOOS JOURNAL OF AUTISM AND DEVELOPMENTAL DISORDERS COMPIEMENTARY THERAPIESIN CLINICAL PRACTICE JOURNAL OF BODYWORK AND MOVEMENT THERAPIES PHYSICAL I\& OCCUPATIONAL THERAPY IN PEDIATRICS REHABILITATION ERICAN JOURNAL OF OCCUPATIONAL THERAPY FRONTIERS IN VETERINARYS

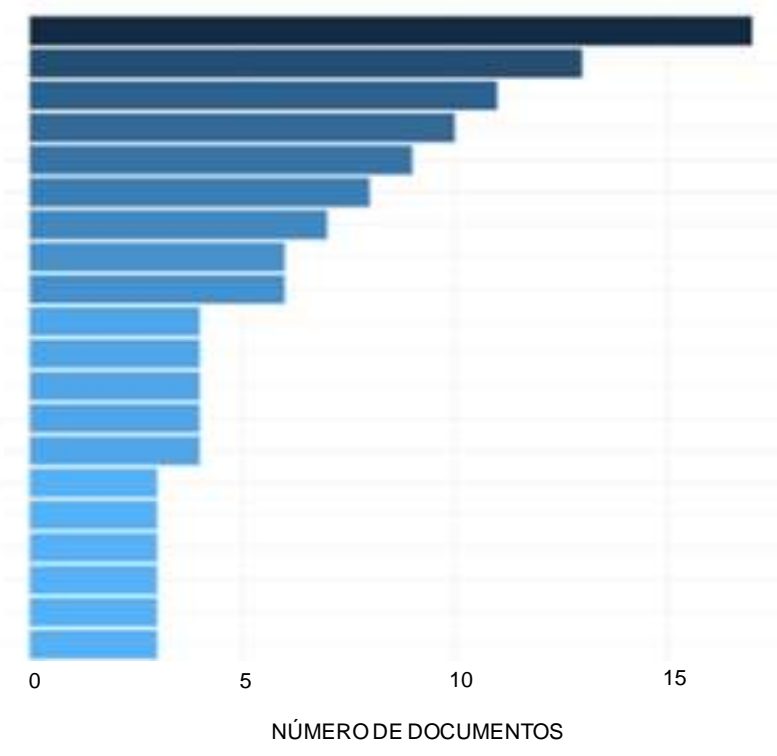

Fonte: Autores (2020).

A produção científica anual obteve um grande aumento na última década, especialmente nos últimos 5 anos, quando foram publicados um total de 227 estudos (Tabela 2 e Figura 3). 
Research, Society and Development, v. 10, n. 4, e20110413191, 2021

(CC BY 4.0) | ISSN 2525-3409 | DOI: http://dx.doi.org/10.33448/rsd-v10i4.13191

Tabela 2. Publicações anuais,

\begin{tabular}{llll}
\hline Anos & Número de publicações & Anos & Número de publicações \\
\hline 1972 & 1 & 2004 & 3 \\
1977 & 1 & 2007 & 4 \\
1978 & 1 & 2008 & 1 \\
1979 & 3 & 2009 & 8 \\
1981 & 1 & 2010 & 7 \\
1987 & 1 & 2011 & 13 \\
1988 & 1 & 2012 & 17 \\
1991 & 1 & 2013 & 11 \\
1992 & 1 & 2014 & 18 \\
1994 & 2 & 2015 & 36 \\
1998 & 1 & 2016 & 34 \\
1999 & 1 & 2017 & 47 \\
2001 & 1 & 2018 & 55 \\
2002 & 1 & 2019 & 55 \\
2003 & 4 & 2020 & 36 \\
\hline
\end{tabular}

Fonte: Autores (2020).

Figura 3. Representação da produção científica anual.

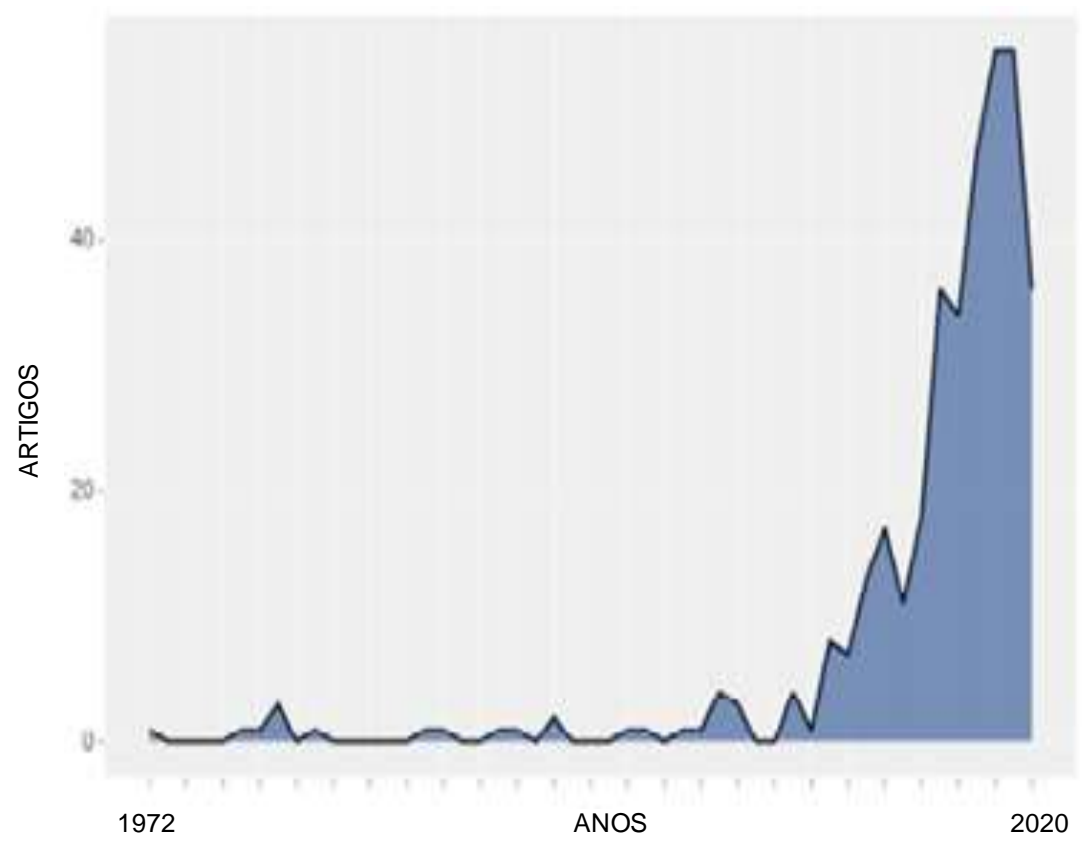

Legenda: Cada pontilhado da vertical corresponde a um intervalo de cinco anos. Fonte: Autores (2020).

O autor mais citado foi ALTIERI, seguido de MCGIBBON e STERBA. A Figura 4 representa, em ordem, os 25 autores mais citados mundialmente. 
Figura 4. Representação dos autores mais citados.

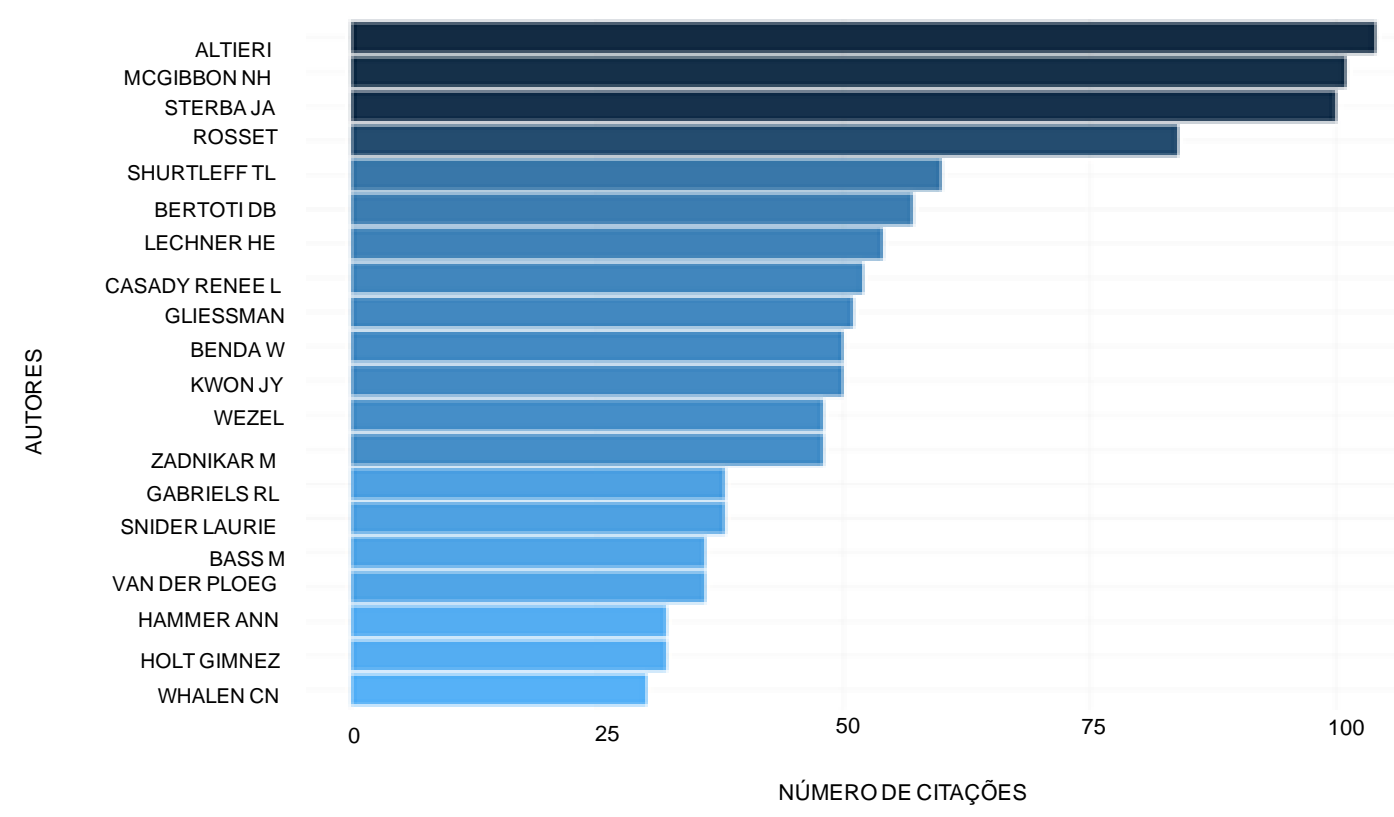

Fonte: Autores (2020).

Quanto à relevância de autores, KIM foi considerado o mais relevante, seguido de LEE e ROSSET. A Figura 5 representa, em ordem, os 20 autores mais relevantes de acordo com o número de publicações globais.

Figura 5. Autores mais relevantes de acordo com o número de publicações.

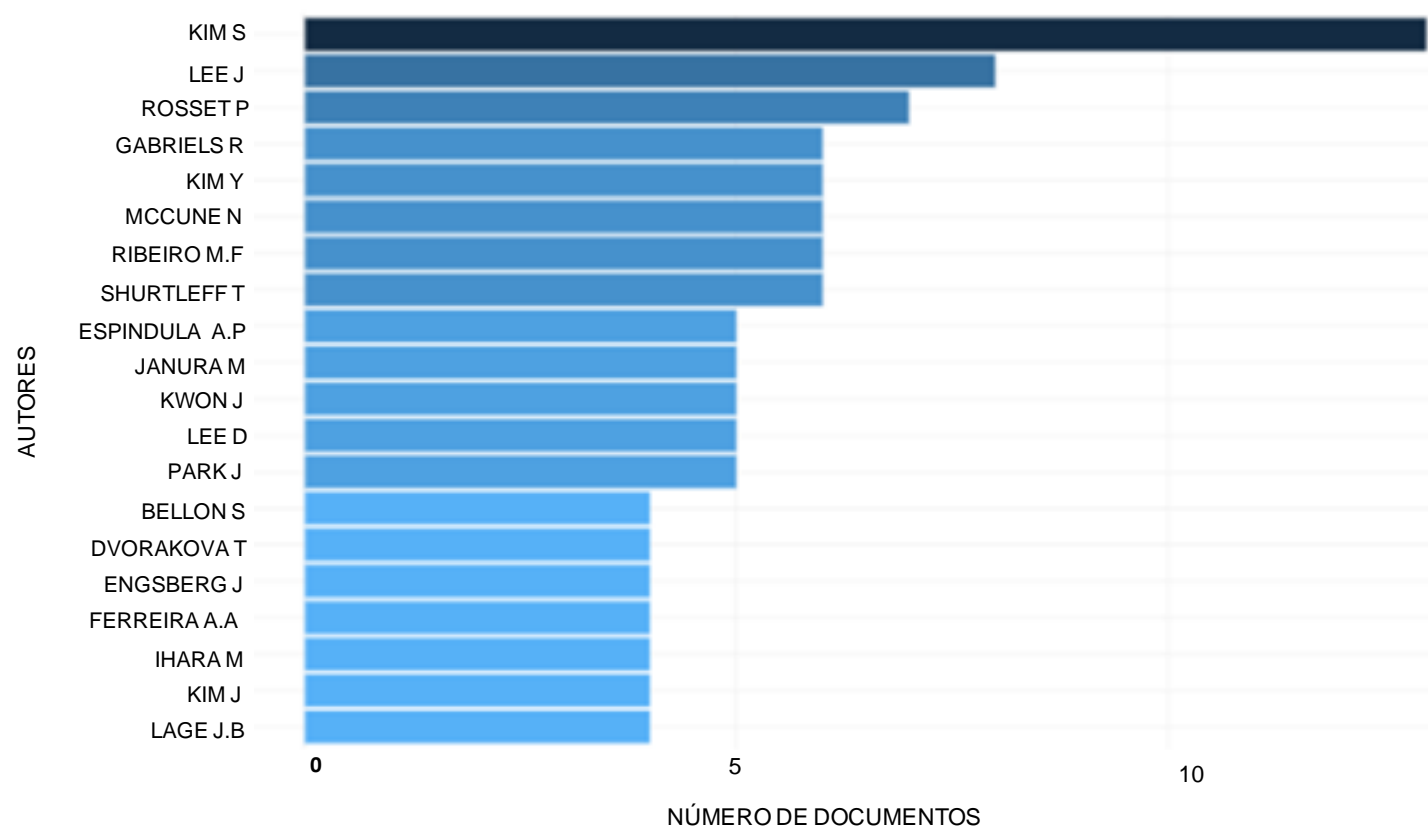

Fonte: Autores (2020).

O estudo intitulado: "Effect of an equine-movement therapy program on gait, energy expenditure, and motor function in children with spastic cerebral palsy: A pilot study", dos autores Mcgibbon, Andrade, Widener e Cintas, publicado em 1998 na revista Developmental Medicine \& Child Neurology, foi o documento mais citado localmente. Os 20 estudos mais citados localmente estão representados na Figura 6. 
Research, Society and Development, v. 10, n. 4, e20110413191, 2021

(CC BY 4.0) | ISSN 2525-3409 | DOI: http://dx.doi.org/10.33448/rsd-v10i4.13191

Figura 6. Representação dos documentos mais citados localmente.

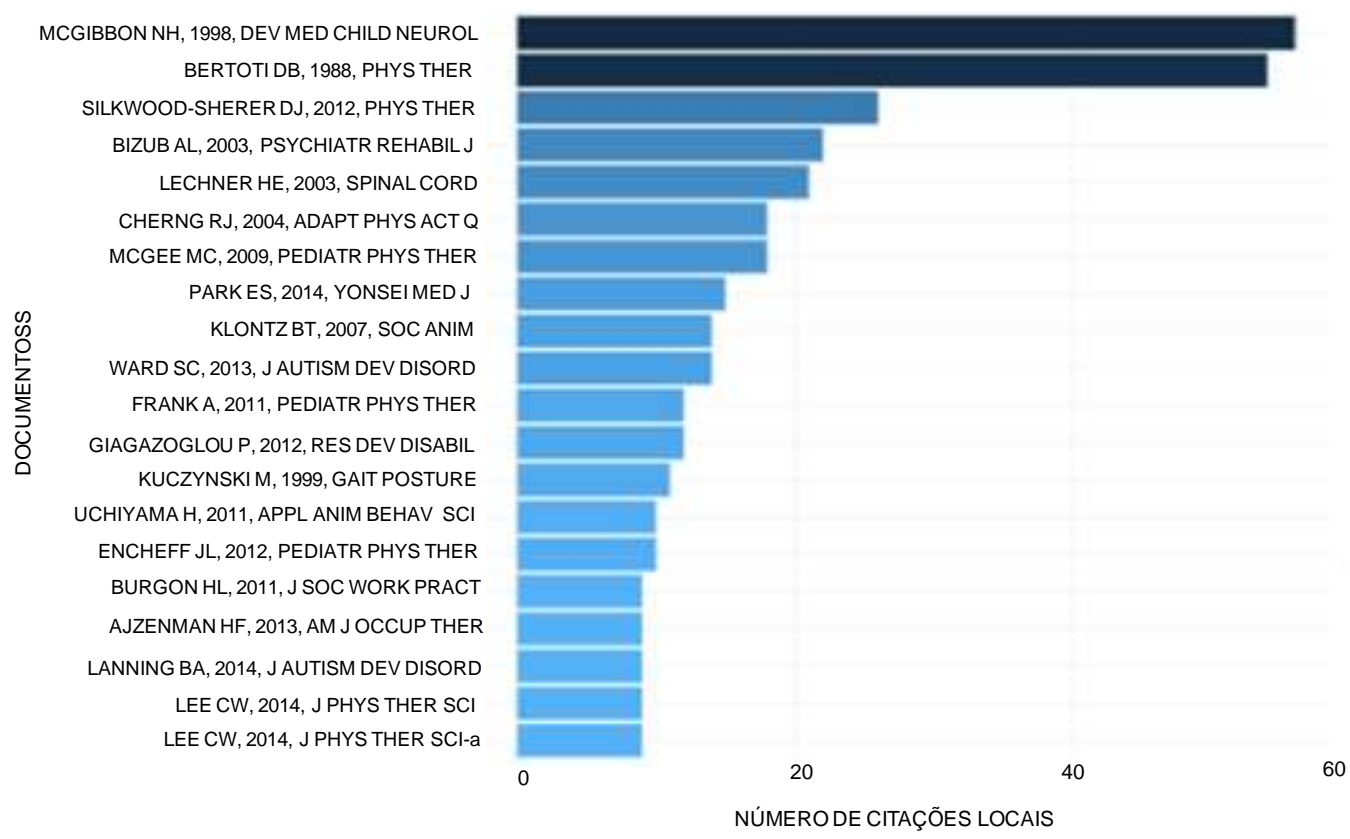

Fonte: Autores (2020).

Já o estudo intitulado: "The effect of therapeutic horseback riding on social functioning in children with autismo", dos autores: Bass, Margaret, Catherine, Duchowny e Maria. Llabre, publicado no Journal of autism and developmental disorders, v. 39, n. 9, p. 1261-1267, 2009, foi o documento mais citado globalmente (Figura 7).

Figura 7. Representação dos documentos mais citados globalmente.

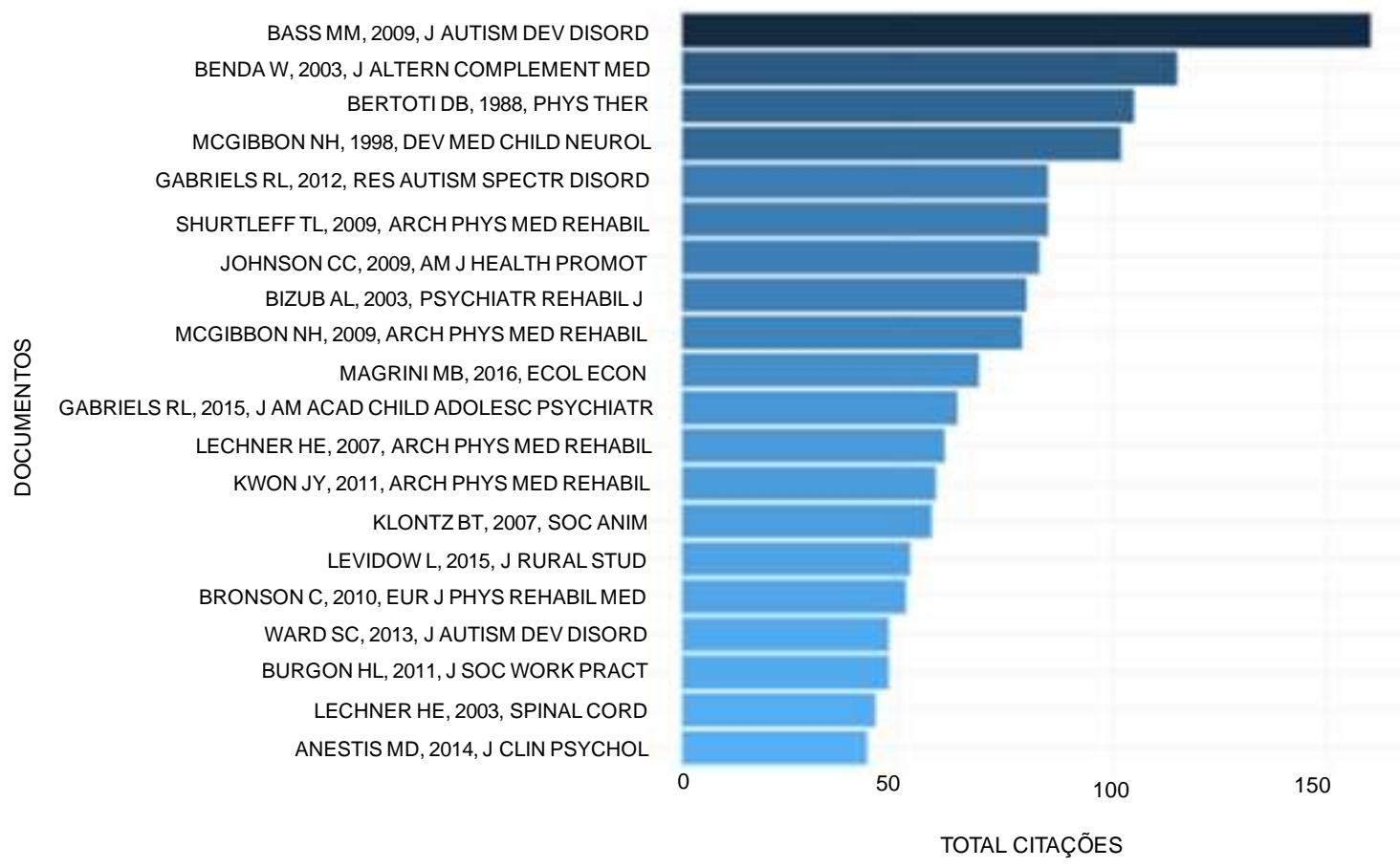

Fonte: Autores (2020). 
A revista Journal of Physical Therapy Science foi considerada o periódico de maior impacto, e o The Journal of Alternative and Complementary; Journal of Peasant Studies e Journal of Autism and Developmental Disorders ocuparam a segunda posição quanto à classificação por fator de impacto. A Figura 8 representa, em ordem de relevância, a classificação de impacto das fontes.

Figura 8. Representação do impacto das fontes levantadas na pesquisa.

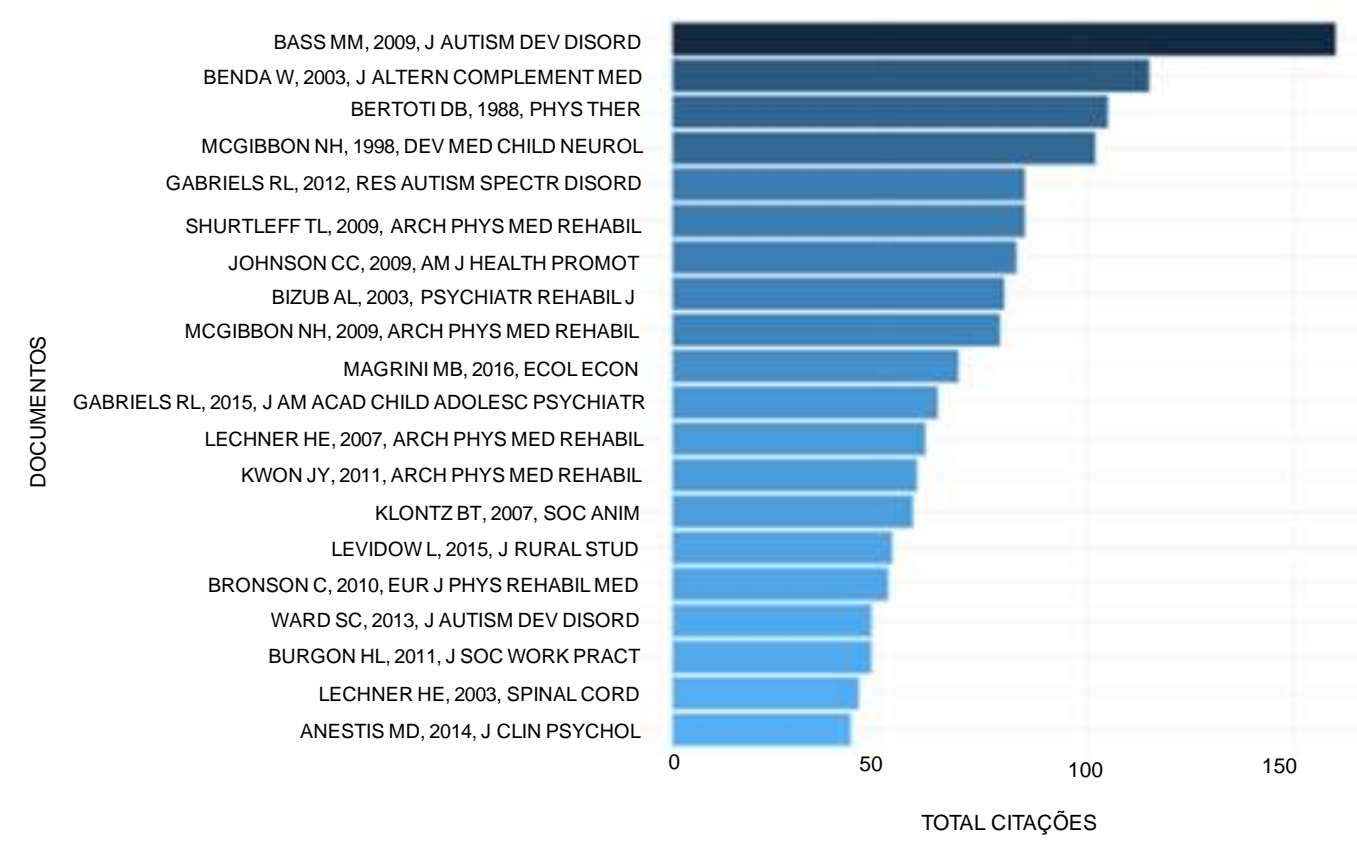

Fonte: Autores (2020).

Dentre os países mais citados, os Estados Unidos ocuparam a primeira posição, seguido da Korea e do Brasil (Figura 9).

Figura 9. Representação dos países mais citados.

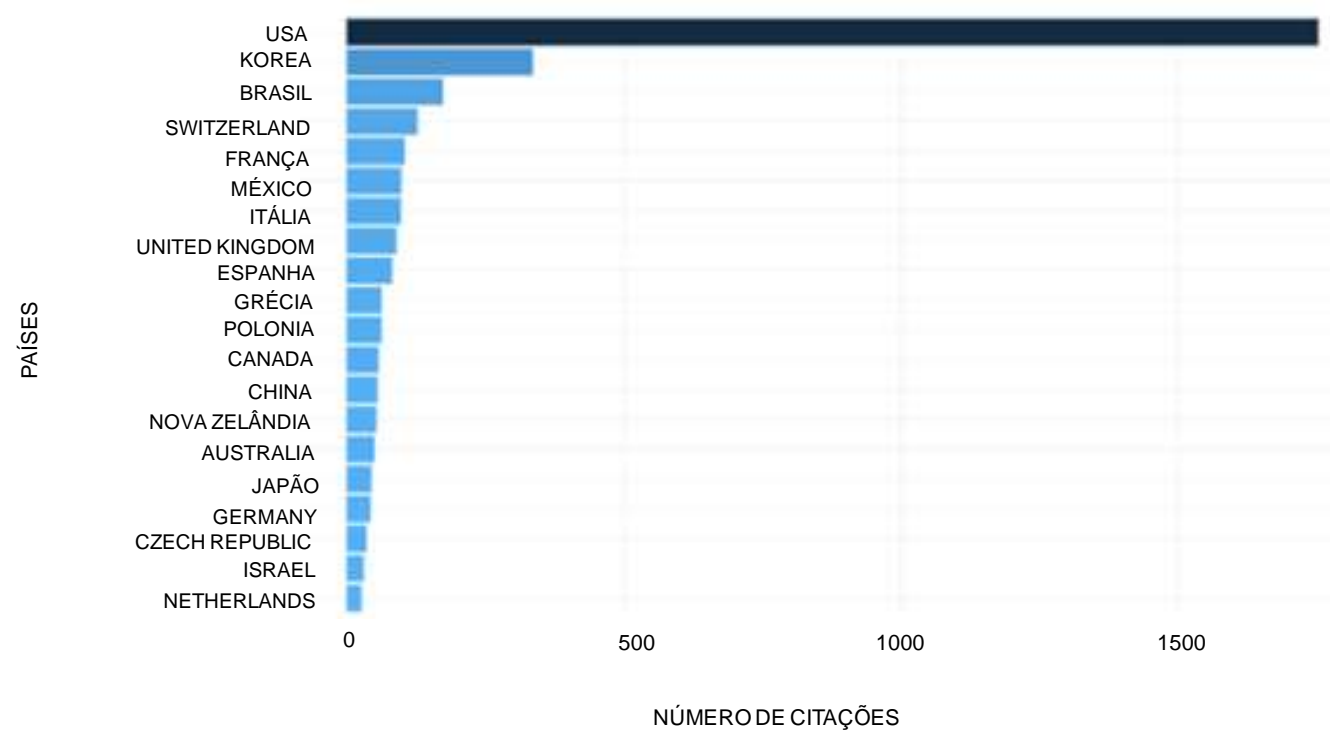

Fonte: Autores (2020). 


\section{Discussão}

O presente estudo teve como objetivo realizar uma análise de mapeamento científico sobre a produção científica na área de equoterapia, durante todo o período, e analisar os principais autores, fontes de publicação e artigos mais citados na área, por meio de uma revisão bibliométrica da ciência na equoterapia e contribuindo com um conjunto amplo de informações científicas sobre a área em questão.

A bibliometria, ferramenta utilizada neste estudo, possibilitou o mapeamento científico por meio da quantificação e estatística para medir índices de produção e disseminação de conhecimento, além de proporcionar a avaliação da qualidade dos periódicos científicos (Lopes et al., 2012) o que possibilitou a aplicação de diversos indicadores bibliométricos, como indicadores de qualidade, fator de impacto e h-index.

Os resultados obtidos mostraram o quanto a pesquisa em equoterapia cresceu nos últimos anos, visto que dos 360 artigos encontrados durante todo o período pesquisado, 1972 (primeiro artigo publicado na área) à 13 de outubro de 2020, 322 estudos foram publicados nos últimos 10 anos, sendo 227 destes nos últimos 5 anos. Esse aumento é relevante visto que o crescimento de publicações científicas fortalece a terapia, o que favorece a continuidade de estudos e o reconhecimento da importância desta modalidade (Zamo \& Zamo, 2012) contribuindo para o crescimento da ciência sobre o método e promovendo melhorias nas práticas clínicas. O aumento da produção científica anual também indica que os estudos sobre o tema estão em constante crescimento e desenvolvimento, o que fortalece a relação entre o conhecimento científico e a tomada de decisão terapêutica. Segundo Marques e Peccin (Marques \& Peccin, 2005) a prática baseada em evidências é uma realidade, devendo ser rotina do profissional basear-se em estudos científicos a fim de conduzir sua intervenção e proporcionar uma terapia atualizada e eficaz.

As palavras chaves mais relevantes, demonstram o quanto a prática da equoterapia relaciona-se com crianças, atendendo as mais diversas síndrome e/ou doenças, em especial, crianças com paralisia cerebral, a fim de proporcionar a melhora da função motora grossa (Ribeiro et al., 2019), do desempenho funcional na vida diária (Park et al., 2014) melhora da marcha e equilíbrio (Kwon, 2011) e do controle postural (Zadnikar \& Kastrin, 2011) tornando-se uma terapêutica ampla e eficaz para essa população.

A Journal of Physical Therapy Science, Revista acadêmica oficial da Society of Physical Therapy Science, publicado pela primeira vez em 1989 (JPTS, 2021), foi considerado a fonte com maior número de publicações e citações sobre a terapia com cavalos. O indicador fator de impacto, usado para a classificação de relevância do periódico, é um dos indicadores de impacto mais conhecidos na comunidade científica (Waltman, 2016) pois trata-se de um método bibliométrico que avalia a importância de periódicos científicos em suas respectivas áreas, que qualifica as revistas por meio da média de citações dos artigos publicados no biênio anterior.

A realização do presente estudo também permitiu uma análise da produção científica durante todo o período, o que contribui para a perspectiva de novos estudos, sendo apresentado os estudos e autores mais citados, o que possibilita a unificação do conhecimento como ferramenta de tratamento e troca de saberes.

O estudo mais citado globalmente, apresentou resultados relevantes sobre o uso do método em crianças com autismo, sendo descrito resultados no aumento da busca e sensibilidade sensorial, motivação social, e diminuição dos comportamentos sedentários (Bass, Catherine \& Llabre, 2009). Tais resultados fornecem evidências para diversos outros estudos decorrentes no assunto, assim como fonte para tomada de decisão clínica para indicação do método terapêutico para essa população.

O estudo mais citado localmente, avaliou por um período de oito semanas a marcha, o gasto energético e a função motora grossa de crianças com paralisia cerebral submetidas ao tratamento da hipoterapia. Os autores observaram tendência de aumento do comprimento da passada e diminuição da cadência. Este estudo sugere que a terapia assistida por cavalos pode 
melhorar o gasto energético durante a caminhada e a função motora grossa em crianças com paralisia cerebral (Mcgibbon, 1998).

$\mathrm{Na}$ análise de países mais citados, os Estados Unidos ocuparam a primeira posição, a Korea a segunda e o Brasil foi considerado como terceiro país mais citado. O primeiro centro de equitação nos Estados Unidos foi criado em 1969, na cidade de Chicago (Andrade \& Augusto, 2007). Em 1969 foi criado a Associação Norte-Americana de Equitação para Deficientes (Narha-North, 2017), atendendo cerca de 30.000 pessoas com deficiências por ano em Denver (EUA). Na Korea, a equitação com fins para a reabilitação foi inserida em 2001, e a Sociedade Coreana de Equitação e Reabilitação fundada em 2013 (KathKorea, 2021). Do mesmo modo, no Brasil a implementação do método ocorreu em 1989, com a fundação da Associação Nacional de Equoterapia - Ande Brasil. Esses resultados mostram o quanto esses países estão se destacando na utilização do método, em especial a Korea, pelo tempo de efetivação da terapia no país.

\section{Considerações Finais}

A análise bibliométrica realizada no presente estudo possibilitou uma revisão da produção científica na equoterapia, sendo esse um estudo inédito, que demonstrou informações relevantes para pesquisadores, acadêmicos e profissionais da área. Observa-se a necessidade de atualizações constantes, pois, apesar de alguns conceitos já estarem bem estabelecidos, novas pesquisas podem trazer informações relevantes para a terapêutica e para o desenvolvimento da ciência. Tais informações beneficiam e ampliam a terapia, o que contribui para a prática baseada em evidências.

Para trabalhos futuros sugere-se que seja realizado estudo de revisão sistemática, integrativa ou da literatura envolvendo os trabalhos relevantes apresentados neste manuscrito.

O presente estudo apresenta importante relevância pois estudos bibliométricos permitem uma análise quantitativa descritiva sobre a ciência em questão, o que promove um mapeamento global da área estudada.

\section{Agradecimentos}

Os autores agradecem o apoio do Conselho Nacional de Desenvolvimento Científico e Tecnológico (CNPq), Coordenação de Aperfeiçoamento de Pessoal de Nível Superior (CAPES), Fundação de Amparo à Pesquisa do Estado de Minas Gerais (FAPEMIG) e ao Núcleo Interdisciplinar de Estudos em Revisões Sistemáticas da UFTM (NIERS).

\section{Referências}

Althobaiti, T., Katsigiannis, S., West, D., \& Ramzan, N. (2019). Examining human-horse interaction by means of affect recognition via physiological signals. IEEE Access, 7, 77857-77867.

Aha. (2021). The hippotherapy methods. American Hippotherapy Association. https://www.americanhippotherapyassociation.org/what-is-hippotherapy.

Ande. (2021). O método equoterapia. Ande-Associação Nacional de Equoterapia.Brasil.http://equoterapia.org.br/articles/index/article_detail/142/2022.

Andrade, M. C. P., \& Augusto, V. (2007). Efeitos da utilização do cavalo como recurso terapêutico na motricidade de crianças portadoras de mielomeningocele. Revista Ciência do Unfifae. 1 (1), 28-35.

Aria, M., \& Cuccurullo, C. (2017). Bibliometrix: An R-tool for comprehensive science mapping analysis. Journal of informetrics. 11 (4), $959-975$.

Baldam, R. (2021). Science Mapping (Bibliometria) with R Studio, Bibliometrix and international indexes. Disponible: http://cope.ufes.br.

Bass, M. M., Catherine, A. D., \& Llabre, M. M. (2009). The effect of therapeutic horseback riding on social functioning in children with autismo". Journal of autism and developmental disorders. 39 (9), 1261-1267.

Campos, C. S. (2007). Equoterapia - O Enfoque Psicoterapêutico com Crianças Down. Departamento de Psicologia. Monografia (Graduação) Universidade Católica de Goiás, Goiânia.

De Mello, E. C., Regalo, S. C. H., Diniz, L. H., Lage, J. B., Ribeiro, M. F., Bevilacqua Junior, D. E., Rosa, R. C., Ferreira, A. A., Ferraz, M. L. F., Teixeira, . P. A., \& Espindula, A. P. (2020). Electromyographic analysis of stomatognathic muscles in elderly after hippotherapy. PLoS ONE. 15 (8), 1-9. 
Diniz, L. H., De Mello, E. C., Ribeiro, M. F., Lage, J. B., Bevilacqua Júnior, D. E., Ferreira, A. A., Ferraz, M. L. F., Teixeira, V. P. A., \& Espindula, A. P. (2020). Impact of hippotherapy for balance improvement and flexibility in elderly people. Journal of Bodywork and Movement Therapies. 24(2) 92-97.

Espindula, A. P., Ribeiro, M. F., Sande de Souza, L. A. P., Ferreira, A. A., Ferraz, M. L. F., \& Teixeira, V. P. A. (2016). Efeitos da equoterapia na postura de indivíduos com Síndrome de Down. Fisioterapia e Movimento. 29(3), 497-506.

JPTS. (2021). The Journal Factor impacto. Journal of Physical Therapy Science https://academic-accelerator.com/Impact-Factor-IF/pt/Journal-of-PhysicalTherapy-Science.

Kath-Korea. (2021). The hippotherapy methods. Association of Therapeutic Horsemanship. http://kath.kr/html/pmode=Introduce.

Koca, T. T., \& Ataseven, H. (2016). What is hippotherapy? The indications and effectiveness of hippotherapy. Northern clinics of Istanbul. 2 (3), 247.

Kwon, J. Y. C. (2011) Effects of hippotherapy on gait parameters in children with bilateral spastic cerebral palsy. Archives of physical medicine and rehabilitation. 92 (5), 774-779.

Lopes, S., Costa, M. T, Fernández-Llimós, F., Amante, M. J., \& Lopes, P. F. (2012). A Bibliometria e a Avaliação da Produção Científica: indicadores e ferramentas. In: Actas do congresso Nacional de bibliotecários, arquivistas e documentalistas.

Marques, A. P., \& Peccin, M. S. (2005). Pesquisa em fisioterapia: a prática baseada em evidências e modelos de estudos. Fisioterapia e pesquisa. 11(1), 43-48.

Matusiak-wieczorek, E. E., Dziankowska-zaborszczyk, E., \& Borowski, M. S. S. (2020). The Influence of Hippotherapy on the Body Posture in a Sitting Position among Children with Cerebral Palsy. International journal of environmental research and public health. 17(18), 6846.

Mcgibbon, N. H., Andrade, C. K., Widener, G., \& Cintas, H. L. (1998). Effect of an equine-movement therapy program on gait, energy expenditure, and motor function in children with spastic cerebral palsy: A pilot study, Developmental Medicine \& Child Neurology. 40(11), 754-62.

Narha-North. (2017). The hippotherapy methods. American Riding for the Handicapped Association, Inc. https://www.nchpad.org/Directories/Organizations/2107/North American Riding for the Handicapped Association Inc NARHA.

Pálsdóttir, A. M., Gudmundsson, M., \& Grahn, P. (2020). Equine-Assisted Intervention to Improve Perceived Value of Everyday Occupations and Quality of Life in People with Lifelong Neurological Disorders: A Prospective Controlled Study. International Journal of Environmental Research and Public Health. 17 (7), 2431.

Park, E. S., Wook Rha, D., Shin, J. S. S., \& Jung, S. (2014). Effects of hippotherapy on gross motor function and functional performance of children with cerebral palsy. Yonsei medical journal. 55(6), 1736-1742.

Path-Learn. About Eaat. (2020). Professional Association of Therapeutic Horsemanship International. https://www.pathintl.org/resourceseducation/resources/eaat/193-eaat-definitions\#hippotherapy.

Pereira, A. S., Shitsuka, D. M., Parreira, F. J., \& Shitsuka, R. (2018). Metodologia da pesquisa científica. UFSM.https://repositorio.ufsm.br/bitstrea $\mathrm{m} /$ handle/1/15824/Lic_Computacao_Metodologia-Pesquisa-Cientifica.pdf?sequence=1

Ribeiro, M. F., Espindula, A. P., Lage, J. B., Bevilacqua Júnior, D. E., Diniz, L. H., De Mello, E. C., Ferreira, A. A., Ferraz, M. L. F., \& Teixeira, V. P. A. (2019) Analysis of the electromiographic activity of lower limb and motor function in hippotherapy practitioners with cerebral palsy. Journal of Bodywork and Movement Therapies. 23 (1), 39-47.

Russell, E. (2013). Horses as healers for veterans. Canadian Medical Association or its licensors. 185(14), 1205.

Waltman, L. A. (2016). Review of the literature on citation impact indicators. Journal of Informetrics. 10 (2), 365-391.

White-lewis, S., Johnson, R., Ye, S., \& Russell C. (2019). An equine-assisted therapy intervention to improve pain, range of motion, and quality of life in adults and older adults with arthritis: A randomized controlled trial. Applied Nursing Research. 49 (1), 5-12.

White-Lewis, S. (2020). Equine-assisted therapies using horses as healers: A concept analysis. Nursing Open. 7(1), 58-67.

Zadnikar, M. \& Kastrin, A. (2011). Effects of hippotherapy and therapeutic horseback riding on postural control or balance in children with cerebral palsy: a meta-analysis. Developmental medicine \& child neurology. 53 (8), 684-69.

Zamo, C. G. S., \& Zamo, R. S. (2012). Bioética, ética e equoterapia https://alfabetizarvirtualtextos.files.wordpress.com/2012/04/bioc3a9tica-c3a9tica-eequoterapia.pdf. 